\title{
BMJ Open Effects of mHealth on the psychosocial health of pregnant women and mothers: a systematic review
}

\author{
Jennifer Lisa Sakamoto (D) , ${ }^{1}$ Rogie Royce Carandang (D) , ${ }^{1}$ Madhu Kharel (1) , \\ Akira Shibanuma (D) , ${ }^{1}$ Ekaterina Yarotskaya, ${ }^{2}$ Milana Basargina, ${ }^{3}$ \\ Masamine Jimba (D) ${ }^{1}$
}

To cite: Sakamoto JL, Carandang RR, Kharel M, et al. Effects of mHealth on the psychosocial health of pregnant women and mothers: a systematic review. BMJ Open 2022;12:e056807. doi:10.1136/ bmjopen-2021-056807

- Prepublication history and additional supplemental material for this paper are available online. To view these files, please visit the journal online (http://dx.doi.org/10.1136/ bmjopen-2021-056807).

Received 06 September 2021 Accepted 24 January 2022

Check for updates

(C) Author(s) (or their employer(s)) 2022. Re-use permitted under CC BY-NC. No commercial re-use. See rights and permissions. Published by BMJ.

${ }^{1}$ Department of Community and Global Health, Graduate School of Medicine, The University of Tokyo, Tokyo, Japan

${ }^{2}$ National Medical Research

Center for Obstetrics, Gynecology and Perinatology

Named after Academician

V.I.Kulakov of the Ministry

of Health of the Russian

Federation, Moscow, Russian

Federation

${ }^{3}$ Department of Neonatal Pathology, National Medical Research Center for Children's Health, Moscow, Russian Federation

Correspondence to Jennifer Lisa Sakamoto; jlsakamoto@m.u-tokyo.ac.jp

\section{ABSTRACT}

Objective To investigate the roles of mobile health, or mHealth, in the psychosocial health of pregnant women and mothers.

Methods A systematic search was conducted in databases and grey literature including MEDLINE, Web of Science, CINAHL, PsycINFO, PsycARTICLES, Academic Search Complete, SocINDEX, Central Register of Controlled Trials, The Database of Abstracts of Reviews of Effects, NHS Economic Evaluation Database, Health Technology Assessment, UNICEF and WHO databases. Two searches were conducted to include original research articles published in English until 15 November 2021. Several tools were used to assess the risk of bias: revised Cochrane risk of bias tool for randomised trials, Risk of Bias in Non-randomized Studies of Interventions, National Heart, Lung, and Blood Institute quality assessment tool for cohort and cross-sectional studies, Critical Appraisal Skills Program checklist for qualitative studies and Mixed Methods Appraisal Tool for mixed-methods studies. Certainty of evidence was assessed using Grading of Recommendations Assessment, Development and Evaluation approach. Due to the high heterogeneity and variability of the included studies, data synthesis was conducted narratively.

Results 44 studies were included among 11999 identified articles. Most studies reported mixed findings on the roles of mHealth interventions in the psychosocial health of pregnant women and mothers; mHealth improved self-management, acceptance of pregnancy/motherhood and social support, while mixed results were observed for anxiety and depressive symptoms, perceived stress, mental well-being, coping and self-efficacy. Furthermore, pregnant women and mothers from vulnerable populations benefited from the use of mHealth to improve their psychosocial health.

Conclusions The findings suggest that mHealth has the potential to improve self-management, acceptance of pregnancy/motherhood and social support. mHealth can also be a useful tool to reach vulnerable pregnant women and mothers with barriers to health information and facilitate access to healthcare services. However, the high heterogeneity limited the certainty of evidence of these findings. Therefore, future studies should identify the context under which mHealth could be more effective.

\section{Strengths and limitations of this study}

- The current study comprehensively reviewed evidence on the roles of mobile health in the psychosocial health of pregnant women and mothers by including multiple domains of psychosocial health outcomes.

- The study followed Preferred Reporting Items for Systematic Review and Meta-Analysis Protocols guidelines and Synthesis Without Meta-analysis guidelines.

- The high heterogeneity and uncertainty across the studies regarding the setting, study design and outcome measures make it difficult to draw firm conclusions

- Only English databases and articles were included in the review and may have limited the interpretation of the study findings.

\section{INTRODUCTION}

Psychosocial health refers to the interrelations of the social environment and psychological health of an individual. ${ }^{12}$ It is multidimensional and covers areas such as depression, stress, self-sufficiency and social support. ${ }^{3}$ During the pregnancy and postpartum period, women are especially vulnerable to facing psychological problems, particularly stress and anxiety disorder with comorbidity of depression. ${ }^{4-6}$ According to a study that systematically reviewed the global prevalence of antenatal and postnatal anxiety, $19.4 \%$ of pregnant women experienced antenatal anxiety across the three trimesters and $13.7 \%$ of mothers experienced postnatal anxiety in the first 6 months following delivery in high-income countries. ${ }^{7}$ In low-income and middle-income countries, the prevalence was significantly higher$34.4 \%$ and $25.9 \%$, respectively. ${ }^{7}$ Experiencing pregnancy and childbirth, especially for the first time, is a drastic transition for women as they grow into the role of becoming a mother. ${ }^{8}$ This psychological ambivalence can 
cause problems for both pregnant women and mothers. Furthermore, psychosocial health among pregnant women is crucial for their well-being and the health of the infant. Anxiety during pregnancy has been associated with adverse effects on infants' and children's development, including premature birth, hyperactivity, cleft lip and impaired brain development. ${ }^{910}$

Using mobile health (mHealth) to deliver pregnancy and postpartum care health services has become more familiar with the advancement of information and communication technologies. mHealth can be delivered through various electronic devices, such as mobile phones, tablet computers, personal digital assistants, and other wearable devices or wireless infrastructure. It is an effective tool to deliver perinatal care interventions because of its cost-effectiveness and scalability, which can benefit both the individual users and the health system. ${ }^{11} 12$ Moreover, delivering interventions via mobile devices is beneficial because of their computational power, portability and price, and the tendency of owners to keep them nearby at all times. ${ }^{13}$ Due to these advancements and the increasing use of mobile phones and the internet, pregnant women and mothers rely on the internet and mHealth applications (apps) to seek sources of health information and services for self-care and child care for a multitude of reasons, such as their desire to connect with other women going through the same experiences and instant professional consultation and reassurance at little or no cost. ${ }^{14}$ mHealth apps can also support pregnant women and mothers to manage their own health, promote a healthy lifestyle, and encourage access to information at any time and place. ${ }^{15}$

Despite the potentials of mHealth, there are also its challenges that remain to be tackled. In low-resource settings, potential barriers to mHealth interventions include the limited level of literacy, access to mobile data, knowledge of technology, cultural beliefs and availability of mobile devices. ${ }^{16}$ The culture and cultural beliefs surrounding the women may have restricted opportunities to learn about technology and therefore limited their skills to navigate mobile services. Other barriers include unstable power supply and poor infrastructure and connectivity to internet, especially in rural or conflictaffected areas. ${ }^{17} 18$ Furthermore, due to the variability in the quality of mHealth services, pregnant women's and mothers' distrust and worry on the security issues and lack of evidence-based information provided to them could also act as a barrier in mHealth interventions. ${ }^{19}$

A study by Dol et al systematically reviewed the impact of mHealth interventions during the perinatal period on maternal psychosocial health outcomes. ${ }^{20}$ The findings suggest that mHealth interventions for supporting breastfeeding and newborn care practices that could improve perceived social support and interventions targeting postpartum depression had an impact in reducing postpartum depression. However, the review included only four psychosocial health outcomes: self-efficacy, social support, anxiety and depression. Although they are considered as common psychosocial health outcomes, other aspects of psychosocial health, such as perceived stress and coping that are often experienced during the perinatal period, should also be considered. Moreover, the review included studies with either a quasiexperimental or randomised controlled study design and focused exclusively on high-income countries. They may have missed valuable information on the advantages and disadvantages of mHealth interventions among pregnant women and mothers that could be observed only through observational and qualitative studies. In addition, it makes it challenging to comprehensively understand the global situation of mHealth interventions in the field of maternal and child health when excluding studies from low-income and middle-income countries.

Furthermore, several other systematic reviews and meta-analyses have also investigated the effectiveness of mHealth on pregnancy and postpartum care. The findings showed that using mHealth to support pregnancy and postpartum care was feasible and appropriate. However, the reviews focused on the roles of mHealth in clinical/health outcomes ${ }^{14}{ }^{21}$ lifestyle behaviours ${ }^{22}$ or the specific perinatal period. ${ }^{23}$ Some reviews were conducted only among either low/middle-income countries or high-income countries ${ }^{24-26}$ or a specific type of mHealth service, such as mobile apps or short messaging services (SMS). ${ }^{14} 152224$ Therefore, this study aimed to review evidence from all studies designs conducted in countries of varying income levels to comprehensively investigate the roles of mHealth in the psychosocial health of pregnant women and mothers.

\section{METHODS}

\section{Patient and public involvement}

Patients and/or the public were not involved in the current systematic review.

\section{Search strategy}

The current systematic review initially followed the Preferred Reporting Items for Systematic Reviews and Meta-Analyses (PRISMA) reporting guidelines (see online supplemental file 1). ${ }^{27}$ It was written in accordance with the registered review protocol on PROSPERO (no. CRD42020188975) (see online supplemental file 2). Eleven online bibliographical databases were searched: MEDLINE, Web of Science, CINAHL, PsycINFO, PsycARTICLES, Academic Search Complete, SocINDEX, Cochrane Central Register of Controlled Trials, The Database of Abstracts of Reviews of Effects, NHS Economic Evaluation Database and the Health Technology Assessment. Grey literature from the UNICEF and WHO databases was also searched. The first two authors (JLS and RRC) developed the search strategy in MEDLINE using a combination of Medical Subject Headings terms and keywords (see online supplemental file 3) and applied no date restriction. The search strategy was improved after using article identification numbers to maximise 
the sensitivity and specificity for identifying relevant articles. The search was conducted at two time points where the initial search was conducted on 31 May 2020 and an updated search on 15 November 2021. Included in the search were original research articles written in English published on or before 15 November 2021. The reference lists of eligible articles were manually searched to screen for additional studies.

\section{Eligibility criteria}

The studies were considered eligible if they described or delivered an mHealth intervention (eg, through mobile apps or SMS) targeted to improve at least one aspect of psychosocial health (eg, depression, stress, anxiety, social support, self-efficacy) among pregnant women and mothers of infants and children aged $0-5$ years. Exclusion criteria were mHealth interventions that (1) were not mobile or tablet based and (2) did not focus on psychosocial health outcomes. We did not include eHealth interventions, such as telemedicine and telehealth interventions that were not exclusively delivered via portable and handheld devices. For example, interventions using telephone for delivering interventions were excluded for the reason that telephones could indicate either cell phones or landline telephones, or both. Eligible study designs included randomised controlled trials (RCTs), quasi-experimental, cohort, observational, cross-sectional and other comparative studies, as well as case studies and evaluation reports. Letters, editorials, reviews, conference abstracts and posters, dissertations and books were excluded. All eligible studies found on the databases were exported to the reference-managing software EndNote to facilitate the study selection process and screen for duplicate records.

\section{Data extraction}

After removing the duplicates, the first two authors (JLS and RRC) screened the titles and abstracts of identified studies for relevance. Next, full-text copies of papers were assessed for eligibility by three authors (JLS, RRC and MK), with any disagreements resolved through discussions; if a consensus was not reached, a fourth author was brought in for discussion at each stage (AS or MJ). Finally, the original three authors (JLS, RRC and MK) extracted data using a standardised extraction form following the Population, Intervention, Comparison and Outcome format on Microsoft Excel to ensure the capture of all necessary information, including title, citation (author, publication, year and source), study area, study objectives, study design, study setting, study population, sample size, types of mHealth interventions, comparison group and summary of reported outcomes.

\section{Risk of bias assessment and certainty of evidence}

After data extraction, three authors (JLS, RRC and MK) independently assessed the risk of bias and methodological rigour of the included studies. Revised Cochrane risk of bias tool for randomised trials (RoB 2.0) developed by Cochrane Collaboration for $\mathrm{RCTs}^{28}$ was used to assess RCT-designed studies. The RoB 2.0 tool comprises a series of signalling questions that elicited information on the features of RCTs relevant to assessing the risk of bias. Once the signalling questions were answered, the RCTs were judged and assigned as low, some concerns or high risk of bias. For non-RCTs, four of the following tools were used for the risk of bias assessment: Risk of Bias in Nonrandomized Studies of Interventions for non-randomised studies of intervention, ${ }^{29}$ National Heart, Lung, and Blood Institute quality assessment tool for cohort and cross-sectional studies, ${ }^{30}$ Critical Appraisal Skills Program checklist for qualitative studies ${ }^{31}$ and Mixed Methods Appraisal Tool for mixed-methods studies. ${ }^{32}$ Any disagreements were settled through discussion to arrive at a consensus among the reviewers. The certainty of the evidence was assessed using the Grading of Recommendations Assessment, Development and Evaluation (GRADE) approach. $^{33}$

\section{Data synthesis}

Due to the broad inclusion criteria, high heterogeneity was observed among the included studies regarding the study designs, measurement tools, statistical analyses and outcomes. Therefore, the current review did not pursue a meta-analysis. Instead, we used Synthesis Without Metaanalysis reporting guidelines (see online supplemental file 4) to conduct data synthesis. ${ }^{34}$ A detailed examination was conducted on the numerical and textual summary findings of the included studies. Findings were then synthesised narratively and the studies were grouped according to psychosocial health outcomes. Summary of findings was presented in a table including psychosocial health outcomes, types of mHealth services, total number the outcome was reported, and whether the finding had no effect, mixed effect, or positive effect. Conclusions were reached in each study for the effects of mHealth intervention. We considered an outcome to have a "positive effect' if the mHealth intervention showed a significant effect (eg, improvement in anxiety/depressive symptoms, increase in mental well-being/self-efficacy) and narrative findings indicated positive results (ie, benefits of using mHealth services). An outcome was considered to have a 'mixed effect' when it showed positive changes but were not necessarily statistically significant (eg, Multidimensional Scale of Perceived Social Support mean score: preintervention 23.3, post-intervention 25.0, $\mathrm{p}=0.80$ ). When there was no significant effect and narrative findings reported negative results, the outcome was considered as 'no effect'.

\section{RESULTS}

\section{Study selection}

A total of 11999 records were identified from all the databases, grey literature and through hand-search at two time points. After removing the duplicates, 11 120 records were retained. Of these, 135 articles were 


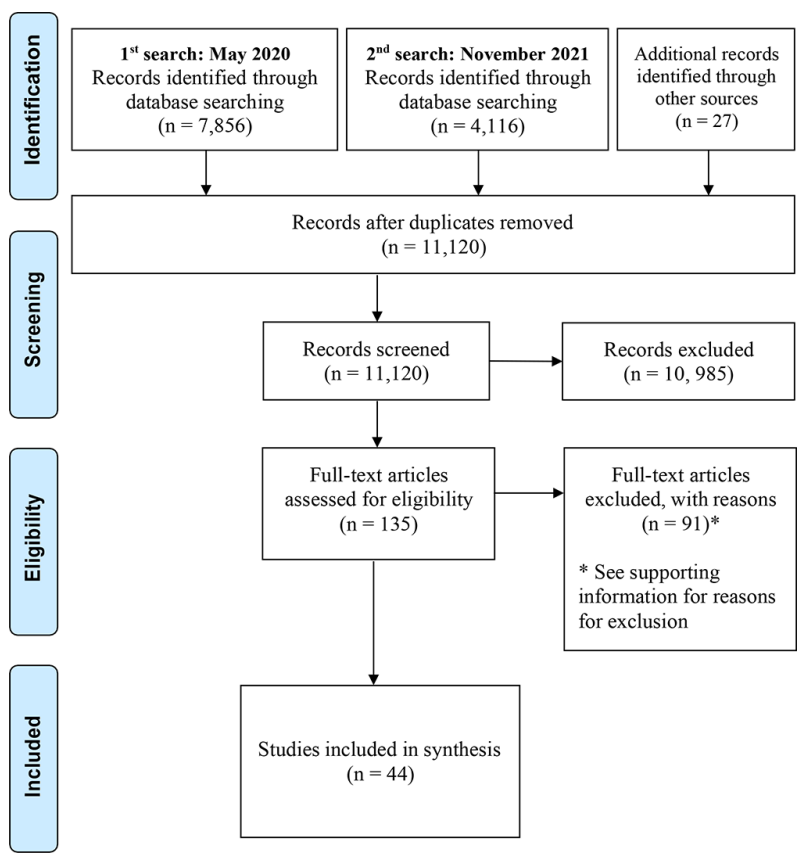

Figure 1 Preferred Reporting Items for Systematic Reviews and Meta-Analyses flow chart.

identified as potential studies for inclusion. The reviewers then assessed the full text of the identified studies and excluded 91 articles (see online supplemental file 5). Finally, a total of 44 articles were included in the final data synthesis. The screening process is depicted in the PRISMA flow diagram (figure 1).

\section{Risk of bias and methodological rigour of included studies}

The risk of bias and the methodological rigour varied across the included studies. Forty-four studies were eligible for the assessment of methodological quality. Out of 44 studies, 17 studies were RCTs ${ }^{35-51}$ and their risk of bias assessment is depicted in figure $2 .{ }^{52}$ Six out of 17 RCTs were assessed as having a high overall risk of bias due to bias arising from randomisation process, deviations from the intended intervention and unclear measurement outcome. ${ }^{38} 394144-46$ Most of the studies were assessed as having some concerns for mainly not being able to blind the participants/outcome assessors due to the nature of the intervention conducted in the studies. ${ }^{35} 37404243475051$ The remaining three studies were considered low risk of bias. ${ }^{36} 4849$

Online supplemental file 6 shows the results of the risk of bias assessment for the non-RCTs. Among the 11 quasi-experimental studies, 4 had a serious risk of bias, ${ }^{53-56} 4$ had a moderate risk ${ }^{57-60}$ and 3 were assessed as low risk. ${ }^{61-63}$ The two observational studies did not have clear information on confounding variables and blinding of the outcome assessors. ${ }^{6465}$ A few studies among the seven qualitative studies ${ }^{66-72}$ lacked information on the positionality of researchers and potential bias may have risen due to the recruitment strategy. Seven mixedmethods study ${ }^{73-79}$ also did not provide any information on confounding. Furthermore, online supplemental file

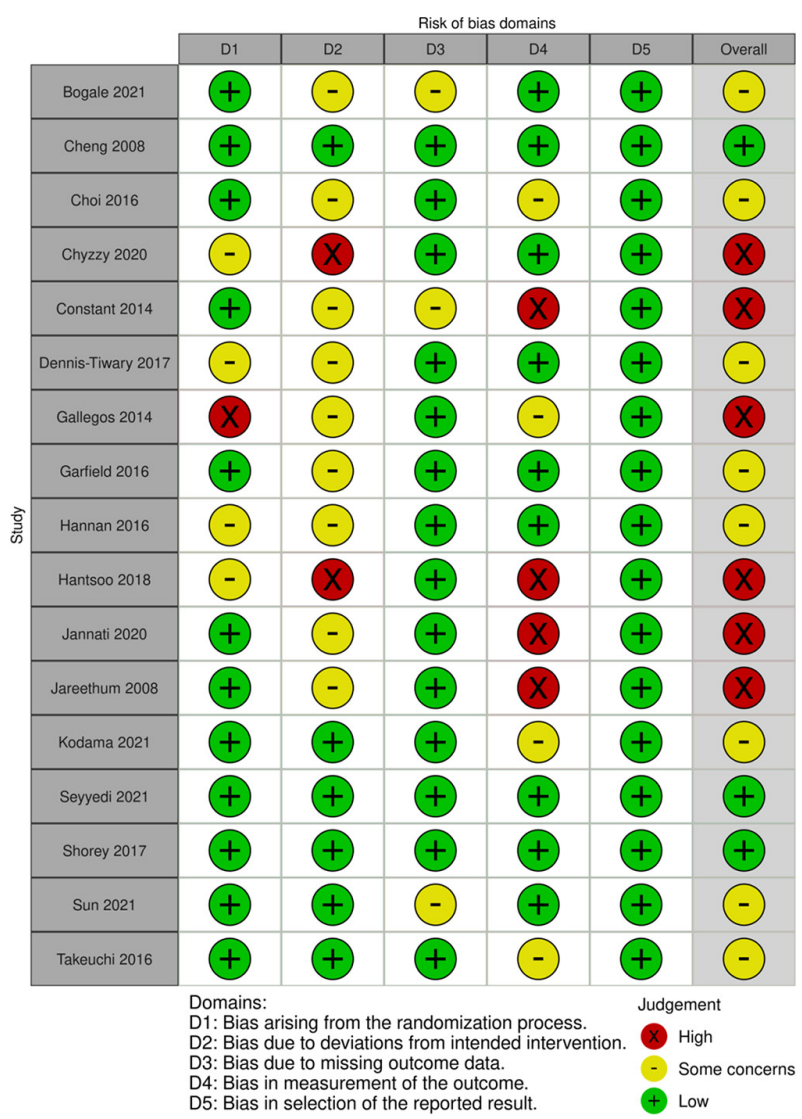

Figure 2 Risk of bias summary for randomised controlled trials based on authors' judgements (low, some concerns, high) about each risk of bias item of the included study.

7 shows the results of the GRADE certainty of evidence assessment.

\section{Study characteristics}

The summary of study characteristics is presented in online supplemental file 8 .

\section{Study countries}

The World Bank income level classification as of June 2021 was incorporated to categorise the countries according to their income level. ${ }^{80}$ Most of the included studies were conducted in high-income countries (31 of 44; 70\%), including the US $(n=15)$, Australia $(n=3)$, Japan $(n=3)$, UK $(n=2)$, Singapore $(n=1)$, Taiwan $(n=1)$, Israel $(n=1)$, Norway $(\mathrm{n}=1)$, Korea $(\mathrm{n}=1)$, Italy $(\mathrm{n}=1)$, Canada $(\mathrm{n}=1)$ and Germany $(\mathrm{n}=1)$. Six studies (6 of $44 ; 14 \%)$ were conducted in upper middle-income countries: South Africa $(n=1)$, Turkey $(n=1)$, Thailand $(n=1)$, China $(n=1)$, Brazil $(n=1)$ and Dominican Republic $(n=1)$. Six studies (6 of 44;14\%) were conducted in lower middle-income countries: Iran $(n=2)$, Kenya $(n=1)$, Zambia $(n=1)$, India $(n=1)$ and Palestine $(n=1)$. Only one study ( 1 of $44 ; 2 \%)$ was conducted in a low-income country, Uganda $(n=1)$.

\section{Study participants}

A slight majority of the studies recruited pregnant women (26 of 44; 59\%) and the recruited population varied from generally healthy pregnant women to at-risk pregnant 
women. Other studies recruited mothers (14 of 44; 32\%), including adolescent mothers and mothers who screened positive for perinatal depression. Few studies recruited both pregnant women and mothers (4 of 44; 9\%). The inclusion criteria for the gestational age of pregnant women and the timeline of the perinatal period varied across studies.

\section{Types of mHealth services and interventions}

There were differences in the mHealth technologies used to facilitate interventions. Twenty-six studies used mobile apps (26 of 44; 59\%) while 11 used SMS (11 of 44; 25\%). Two studies used both SMS and voice calling on mobile phones $(2$ of $44 ; 5 \%)$. A few of the mHealth interventions were delivered using other mHealth services, such as instant messaging services (eg, WhatsApp Messenger) (2 of $44 ; 5 \%$ ), e-learning service via cellphone internet ( 1 of $44 ; 2 \%)$, smartphone website ( 1 of $44 ; 2 \%$ ) and mobilebased interactive voice response system (1 of 44; $2 \%$ ).
The types of mHealth interventions were widely diverse. The interventions were categorised into 10 types incorporating the mHealth and Information and Communications Technology (ICT) Framework ${ }^{81}$ and its adaptation. ${ }^{21}$ Studies were counted in more than one category type if the mHealth intervention included multiple functions. The 10 types include health education or promotion (19 of 44; 43\%), communication and support (15 of 44; $34 \%$ ), psychoeducation or therapy ( 11 of $44 ; 25 \%$ ), selfmonitoring system (6 of 44; 14\%), reminders (4 of 44; $9 \%$ ), decision guideline (3 of 44; 7\%), behaviour change ( 3 of $44 ; 7 \%$ ), laboratory results ( 1 of $44 ; 2 \%$ ), registries/ vital events tracking (1 of 44;2\%) and electronic health records ( 1 of $44 ; 2 \%)$.

\section{Study outcomes}

In total, 77 comparisons were made across the 44 included studies that examined the roles of mHealth interventions in psychosocial health outcomes among pregnant women

Table 1 Summary of the effect of mHealth interventions on psychosocial health outcomes of pregnant women and mothers

\begin{tabular}{|c|c|c|c|c|c|}
\hline $\begin{array}{l}\text { Psychosocial health } \\
\text { outcomes }\end{array}$ & $\begin{array}{l}\text { Types of mHealth } \\
\text { services (n) }\end{array}$ & $\begin{array}{l}\text { No effect, } \\
\text { (n) }\end{array}$ & $\begin{array}{l}\text { Mixed effect, } \\
\text { (n) }\end{array}$ & $\begin{array}{l}\text { Positive effect, } \\
\text { n (\%) }\end{array}$ & $\begin{array}{l}\text { Total number outcome } \\
\text { reported, (n) }\end{array}$ \\
\hline $\begin{array}{l}\text { Anxiety symptoms } \\
(35363940444647505461-6373)\end{array}$ & $\begin{array}{l}\text { SMS (6) } \\
\text { App (7) }\end{array}$ & 3 & 6 & 4 (31) & 13 \\
\hline $\begin{array}{l}\text { Depressive symptoms } \\
(374445495054-5660616370)\end{array}$ & $\begin{array}{l}\text { SMS (1) } \\
\text { App (9) } \\
\text { IVRS (1) } \\
\text { IM (1) }\end{array}$ & 3 & 4 & $5(42)$ & 12 \\
\hline $\begin{array}{l}\text { Mental well-being } \\
\left({ }^{5564}\right)\end{array}$ & $\begin{array}{l}\text { SMS (1) } \\
\text { App (1) }\end{array}$ & 1 & 0 & $1(50)$ & 2 \\
\hline 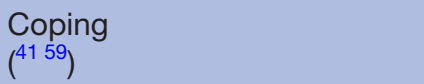 & $\begin{array}{l}\text { SMS (1) } \\
\text { App (1) }\end{array}$ & 1 & 1 & $0(0)$ & 2 \\
\hline
\end{tabular}

(1)

\begin{tabular}{|c|c|c|c|c|c|}
\hline $\begin{array}{l}\text { Self-management } \\
\left({ }^{4471727477)}\right.\end{array}$ & $\begin{array}{l}\text { SMS (1) } \\
\text { App (4) }\end{array}$ & 0 & 0 & $5(100)$ & 5 \\
\hline $\begin{array}{l}\text { Acceptance } \\
(536569)\end{array}$ & $\begin{array}{l}\text { SMS (0) } \\
\text { App (3) }\end{array}$ & 0 & 0 & $3(100)$ & 3 \\
\hline $\begin{array}{l}\text { Social support from partners } \\
(49656875)\end{array}$ & $\begin{array}{l}\text { SMS (1) } \\
\text { App (3) }\end{array}$ & 0 & 0 & 4 (100) & 4 \\
\hline $\begin{array}{l}\text { Social support from } \\
\text { healthcare providers } \\
(557276)\end{array}$ & $\begin{array}{l}\text { SMS (1) } \\
\text { App (2) }\end{array}$ & 0 & 0 & $3(100)$ & 3 \\
\hline $\begin{array}{l}\text { Social support from other } \\
\text { sources } \\
(37384143566769747879)\end{array}$ & $\begin{array}{l}\text { SMS (3) } \\
\text { App (3) } \\
\text { SMS/voice call (2) } \\
\text { IM (2) }\end{array}$ & 1 & 3 & $6(71)$ & 10 \\
\hline
\end{tabular}

app, mobile application; IM, instant messaging service; IVRS, interactive voice response system; mHealth, mobile health; SMS, short messaging service. 
and mothers (see table 1). Some studies assessed multiple psychosocial health outcomes. Therefore, results are reported based on the number of comparisons made for each outcome.

\section{Anxiety symptoms}

Thirteen studies assessed the roles of mHealth in anxiety symptoms among pregnant women and mothers. $35363940444647505461-6373$ Four studies found positive effects, six studies had mixed findings and three studies found no significant effect (GRADE certainty of evidence: low). Jareethum et al conducted an RCT study in Thailand which sent two SMS messages per week containing information and warnings on abnormal symptoms appropriate to the women's gestational age. ${ }^{46}$ As a result, pregnant women who received an SMS had lower anxiety levels during the antenatal and perinatal period than those who did not receive any SMS; however, it was only significant during the antenatal period $(\mathrm{M}=2.78$ vs 4.93, $\mathrm{p}=0.002$ ). Another RCT study conducted by Constant et al in South Africa sent 13 automated text messages with reminders to take medication and information about side effects to women undergoing medical abortion. ${ }^{40}$ Between baseline and follow-up, women who received the messages reported a decrease in their Hospital Anxiety and Depression Scale (HADS) score compared to the control group $(\mathrm{M}=11.40$ vs $7.80, \mathrm{p}=0.013) .{ }^{39}$

A pilot RCT conducted by Dennis-Tiwary et al in the US investigated the effectiveness of an attention bias modification training app to reduce pregnancy threat, anxiety and stress, and did not find any significant changes in the anxiety domain of Depression, Anxiety and Stress Scale between intervention $(\mathrm{M}=3.20, \mathrm{SD}=3.00)$ and control group $(\mathrm{M}=2.07, \mathrm{SD}=3.60)$ at 1 -month follow-up. ${ }^{40}$ They also did not find any change in the Hamilton Anxiety Scale scores between intervention $(\mathrm{M}=9.20, \mathrm{SD}=6.71)$ and control groups $(\mathrm{M}=6.93, \mathrm{SD}=9.10)$. Similarly, Baumel et al conducted a quasi-experimental study to examine the effectiveness of an app that provided self-help tools and emotional support delivered by trained volunteers to pregnant women diagnosed with postpartum depression. They found no significant changes in the Beck Anxiety Inventory (BAI) scores between baseline $(\mathrm{M}=20.47$, $\mathrm{SD}=13.15)$ and at 1-month follow-up $(\mathrm{M}=16.65, \mathrm{SD}=7.52$, $\mathrm{p}=0.11){ }^{61}$

\section{Depressive symptoms}

Twelve studies assessed the roles of mHealth in self-reported levels of depressive symptoms. ${ }^{374445495054-5660616370}$ Five studies found a positive effect, four studies had mixed findings and three studies found no significant effect (GRADE certainty of evidence: low). Song et al conducted a quasi-experimental study to test the effectiveness of a two-way text-messaging system to distribute health-related information to pregnant women with low socioeconomic status living in the US. They reported a reduction in the Center for Epidemiological Studies Scale score after the intervention $(t(19)=2.991, \mathrm{p}<0.01) .^{55}$
Mixed results were reported among studies assessing the roles of mHealth in improving depressive symptoms using the Edinburgh Postnatal Depression Scale (EPDS). In Singapore, Shorey et al examined the effectiveness of a psychoeducational app to improve parenting outcomes. Parents in the intervention group reported no significant difference in the EPDS scores compared with the control group (Mean difference $=7.00$ vs 7.60).${ }^{49}$ Similarly, Dalton et al reported no changes in EPDS scores between intervention and control group (detailed results of analyses not reported) and when comparing pre-intervention and post-intervention $(\mathrm{M}=6.08$ vs $5.66, \mathrm{p}=0.635) .{ }^{54}$ However, Baumel et al reported significant decline in EPDS scores after conducting an intent-to-treat analysis from baseline $(\mathrm{M}=17.32, \mathrm{SD}=5.96)$ and after 30 -day follow-up $(\mathrm{M}=13.53$, $\mathrm{SD}=4.65, \mathrm{p}=0.005)$. Beck Depression Inventory II scores also significantly improved $(\mathrm{M}=26.11, \mathrm{SD}=13.34 ; \mathrm{M}=19.18$, $\mathrm{SD}=9.23, \mathrm{p}=0.01){ }^{61}$

\section{Perceived stress}

Perceived stress was assessed in seven studies. 39404350555960 Three studies found positive effects, two studies had mixed findings and two studies found no significant effect (GRADE certainty of evidence: very low). Constant et al reported lower scores on the Impact of Event ScaleRevised, which indicated lower levels of emotional stress $(\beta=-1.8,95 \% \mathrm{CI}=-3.2$ to $-0.4, \mathrm{p}=0.015)$ among the intervention group than those in the control group when adjusted for baseline anxiety. ${ }^{39}$ Furthermore, Jallo et al conducted a quasi-experimental study to investigate the effectiveness of a stress coping app to reduce stress in a sample of pregnant women staying in an obstetrical antepartum high-risk unit. They reported an immediate drop in their Visual Analog Stress Scale score when comparing before and after listening to the app with guided imagery audio ( $\mathrm{M}=44.13$ vs $22.04, \mathrm{p}<0.0001)$. However, no differences were found when comparing Perceived Stress Scale scores between pre-intervention and post-intervention (median score $=22.0$ vs $22.0, \mathrm{p}=0.750) .{ }^{59}$

\section{Mental well-being}

Two studies assessed mental well-being. ${ }^{55} 64$ One study found a positive effect, while one study did not find any significant effect (GRADE certainty of evidence: very low). Song et al reported improvement in RAND Mental Health Inventory scores between pre-intervention and post-intervention $(t(19)=-4.241, \mathrm{p}<0.001) .{ }^{55}$ However, Deave $e t$ al reported no significant difference in WarwickEdinburgh Mental Well-Being Scale score between app and non-app users (median score $=54.5$ vs $55, \mathrm{p}=0.284$ ) ${ }^{64}$

\section{Coping}

Coping outcomes were assessed in two studies; one study reported mixed findings, while one study reported no significant effect (GRADE certainty of evidence: very low). ${ }^{41}{ }^{59}$ Gallegos et al assessed the role of mHealth in coping among breastfeeding Australian mothers. The automated text message asked the mothers about 
their breastfeeding experience, and if their responses expressed some level of distress, a breastfeeding counsellor contacted the woman. As a result, mothers expressed higher levels of active coping $(\mathrm{p}=0.01)$ and lower levels of emotion-focused coping $(\mathrm{p}=0.001)$ on the Ways of Coping Checklist. ${ }^{41}$ However, no significant change in the Coping Self-Efficacy Scale scores was reported between pre-intervention and post-intervention in the study conducted by Jallo et al (median score $=148.5$ vs $155, \mathrm{p}=0.129) .{ }^{59}$

\section{Self-efficacy}

Self-efficacy outcomes were assessed in 16 studies. ${ }^{3741} 4246484951535457586466697178$ Seven studies found positive effects, two studies had mixed findings and seven studies found no significant effect (GRADE certainty of evidence: low). Positive findings were often mentioned during qualitative interviews. Adolescent mothers in the US described a sense of fulfilment, competence and confidence from interacting with text messages, which validated their motherhood role. ${ }^{66}$ A formula feeding mother in Australia also described a sense of enhanced confidence by using the app. ${ }^{69}$

However, some quantitative studies reported no change in self-efficacy. Deave et al did not report any change in the Tool to Measure Parenting Self-Efficacy score between baseline and 3-month follow-up (adjusted OR=1.12, 95\% $\mathrm{CI}=0.59$ to $2.13, \mathrm{p}=0.730) .{ }^{64}$ Moreover, breastfeeding mothers reported no changes in the Breastfeeding Self-Efficacy Scale score between baseline and 2-month follow-up ( $\mathrm{M}=4.00$ vs 4.15$)$ and no significant differences in change over time between intervention and control group $(p=0.25){ }^{41}$

\section{Self-management}

Self-management was assessed in five studies ${ }^{44} 71727477$ of which all reported positive findings (GRADE certainty of evidence: low). Hantsoo et al reported that the intervention group rated their ability to manage their health significantly better than the control group $(\mathrm{F}=4.03$, $\mathrm{df}=4$ and $49, \mathrm{p}=0.007)$ at 8 -week follow-up. ${ }^{44}$ Blackwell et al conducted a mixed-methods study in the US and reported that the proportion of minority immigrant pregnant women likely to strongly agree that the text messages allowed them to have greater control over their prenatal healthcare increased from pre-intervention to post-intervention ( $28.6 \%$ vs $51 \%, \mathrm{p}=0.02) .{ }^{74}$ In Norway, women with gestational diabetes mellitus who used an app described an increase in feeling of control to manage their own health: 'I felt that to record [information] in the app was very important.... In that way the app was very important because it gave me a feeling of control' (p. 105). ${ }^{71}$

\section{Acceptance}

Three studies assessed positive outcomes of acceptance regarding pregnancy and motherhood (GRADE certainty of evidence: very low) ${ }^{536569}$ In Turkey, Özkan Şat et al reported in the mean subscale score of Prenatal Self Evaluation Questionnaire (PSEQ) that pregnant women who used apps had a better adaptation level to pregnancy than those who did not use any apps $(\mathrm{M}=18.99$ vs 20.86, $\mathrm{p}=0.005) .{ }^{65}$ Litterbach et al reported that the app reassured infant-feeding mothers of their feeding decisions and help accept that they were doing the 'right thing' for their baby. ${ }^{69}$

\section{Social support from partners}

Partner social support outcomes were assessed in four studies, all presenting positive findings (GRADE certainty of evidence: very low). ${ }^{496568} 75$ In Kenya, Harrington $e t$ al conducted a qualitative study and found that pregnant women and mothers who received text messages on family planning felt improved communication with their partners, which allowed them to start a conversation about family planning. ${ }^{68}$ Shorey et al reported that parents showed a significant difference in the Perceived Social Support for Parenting score from their spouses compared with the control group who did not use the app (mean difference $=27.08,95 \%$ CI $=20.94$ to 34.80 , $\mathrm{p}<0.001) .{ }^{49}$ Pregnant women in Turkey who used apps reported lower mean subscale score for the relationship with their husband on the PSEQ compared with those who did not use apps $(M=13.28$ vs $15.69, p=0.001) .{ }^{65}$

\section{Social support from healthcare providers}

Three studies examined the roles of mHealth intervention in providing social support from healthcare providers (GRADE certainty of evidence: very low). 557276 Sixty per cent of low-income pregnant women reported that the two-way SMS encouraged them to put forward more questions to discuss with their healthcare providers and they felt more prepared to see their healthcare provider. ${ }^{55}$ Pregnant women with diabetes who received informational, motivation and logistical messages via SMS reported that they felt more connected with their healthcare providers. ${ }^{72}$

\section{Social support from other sources}

Ten studies reported on the roles of mHealth in social support from other sources (eg, family, friends and online community). ${ }^{37384143566769747879}$ Six studies found a positive effect, while three reported mixed findings and one study reported no significant effect (GRADE certainty of evidence: low). Litterbach et al found that the app gave support to infant-feeding mothers during times of need, such as when they were questioning their milk supply and when it was impossible to seek advice from others (eg, in the middle of the night). Mothers who were formula feeding or mixed feeding indicated that the programme allowed them to receive support without fear of judgement regarding their decision to use formula. ${ }^{69}$ Moreover, Connor et al reported that the app allowed pregnant women and mothers to receive support from the online community through message boards when they could not rely on friends or family. ${ }^{67}$ 


\section{DISCUSSION}

Findings suggest that mHealth interventions had a positive effect on improving self-management of health and acceptance of pregnancy and motherhood. However, it had mixed effects in anxiety and depressive symptoms, perceived stress, mental well-being, coping, and selfefficacy among pregnant women and mothers. Moreover, mHealth interventions had largely a positive effect on social support from partners, healthcare providers and other sources. Pregnant women and mothers from a socially disadvantaged background, having pre-existing health conditions and behaviours, or dealing with sensitive perinatal issues benefited especially from the mHealth interventions.

mHealth interventions improved self-management and acceptance of pregnancy and motherhood among pregnant women and mothers. This finding is new as Dol et $a l^{20}$ did not report the roles of mHealth interventions in neither outcome. The intervention populations included: pregnant women who are minority immigrants, ${ }^{74}$ with low socioeconomic status and having depressive symptoms, ${ }^{44}$ and postpartum women diagnosed with gestational diabetes mellitus. ${ }^{71}$ These findings suggest that mHealth interventions has the potential to improve self-management of health among pregnant women and mothers who may have pre-existing health conditions and living in resource-limited settings. mHealth can provide ease of access to health education materials and self-monitoring systems where the users can track their own behaviour or health data while also being supported by healthcare providers. Furthermore, three studies reported on the positive roles of mHealth interventions in improving acceptance of pregnancy and motherhood. ${ }^{5365}$ In Australia, infant-feeding mothers reported that the mHealth intervention provided reassurance to their feeding decisions. ${ }^{69}$ Infant-feeding mothers could often face difficulties in deciding feeding practices and feel anxious if they made the right decision, especially if they are first-time mothers. mHealth services can potentially provide both informational and emotional reassurance during such decisions.

The use of mHealth largely improved social support from partners, healthcare providers and other sources. This finding is new because Dol et $a t^{20}$ reported that mHealth interventions showed benefits to perceived social support but did not specifically address the source of the social support. A two-way text messaging system in Kenya that provided family planning education to pregnant/postpartum women and their partners improved communication and support between them. ${ }^{68}$ When family planning education messages are sent directly to men, it is easier for women to communicate effectively with their partners about contraception and partners may gain a better understanding from such communication, leading to more positive attitudes and increased use of contraception. ${ }^{82}$ Moreover, although the service is not provided in person, mHealth could provide pregnant women and mothers opportunity to use online forums and join groups where they can share and learn from others' experiences.

mHealth can act as a support system for vulnerable pregnant women and mothers. In South Africa, women undergoing the home phase of medical abortion who received text messages providing timely information on managing their abortion symptoms were more likely to report improved anxiety symptoms than those who did not. ${ }^{39}$ The messages guided the women through the medical abortion process using a supportive tone without overtly addressing negative emotions. Mothers in Australia who received tailored SMS messages about infant feeding reported that the messages provided support without judgement about their decision to use formula. ${ }^{69}$ The mothers were reluctant to discuss formula use with healthcare providers because of the fear of being judged. Young women encountering decisions about abortion and infant feeding are at risk of social judgements and discrimination. ${ }^{83}$ Fear of judgement could prevent women from openly discussing their health concerns and delay help-seeking during the perinatal period. $^{85}$ Findings suggest that mHealth interventions can provide support to pregnant women and mothers who may feel hesitant to seek support in person due to the fear of being judged. The flexible nature of mHealth interventions enables accommodating users' specific needs and tailoring the programme to their preferences (eg, cultural, literacy, language preferences). This makes it particularly useful for vulnerable populations who often have specific physical and mental health needs. ${ }^{86}$ The findings add to the existing literature that mHealth interventions reduce access barriers among populations vulnerable to health disparities. ${ }^{87-89}$

The current systematic review has several limitations. First, high heterogeneity of outcomes was observed among the included studies due to the broad inclusion criteria and search strategy, making it difficult to conduct a meta-analysis. Studies had a wide range of study designs, sample sizes $(n=4$ to $n=2782)$ and outcome measures (eg, anxiety was assessed with Generalized Anxiety Disorder-7, BAI, HADS), which made the comparison of results difficult. Moreover, positive effects may have been emphasised in qualitative studies and studies with small sample sizes may have reported larger effect sizes. ${ }^{14}$ Thus, the findings should be interpreted cautiously. Second, environmental factors, such as neighbourhood environment, were not included in our review scope, which could have been important factors affecting the psychosocial health of the given population. ${ }^{90}$ Third, the current review exclusively searched for articles in English and this may have limited the number of articles identified during our search. Fourth, non-binary terms were not included as search terms and this may have excluded non-binary people from our study. Lastly, the interaction between individual domains of psychosocial health outcomes was not assessed in the current review as it was beyond the scope of the study. Some findings may be a result of the interaction of the outcomes and not necessarily the effect 
of the mHealth intervention. Despite those limitations, this review addresses research gaps concerning mHealth and pregnancy and postpartum care.

Although the advantages of mHealth interventions have been highlighted, some studies discussed their limitations. Pregnant mothers and women who participated in the mHealth interventions may have been actively seeking help ${ }^{44} 46$ and may have come from a well-resourced environment with higher socioeconomic status. ${ }^{39} 49$ Despite the advancement of technology, the digital divide and digital literacy barriers might have prevented socially disadvantaged pregnant women and mothers from participating in the intervention. Moreover, technical difficulties were also reported in a few studies. Such difficulties included operating system limitations (eg, Google's Android or Apple's iOS), ${ }^{54}$ app failure, which reduced engagement, ${ }^{69}$ and answerability of the system, which created frustration for participants. ${ }^{55}$ Concerns for data security were also expressed. ${ }^{67}$ These technical difficulties could have negatively affected the use of mHealth services, ${ }^{91}$ thus affecting health outcomes. Future studies should consider preventing such difficulties for an effective mHealth intervention.

\section{CONCLUSIONS}

mHealth plays a positive role in improving selfmanagement and acceptance of pregnancy and motherhood. It also has the potential to provide social support from partners, healthcare providers and other sources. mHealth interventions were especially crucial in improving the psychosocial health among vulnerable pregnant women and mothers. However, some studies reported mixed findings on the effectiveness of mHealth on psychosocial health outcomes. The high heterogeneity and uncertainty across the studies regarding the setting, study design and outcome measures make it difficult to draw firm conclusions; thus, these findings should be interpreted with caution. Future studies using mHealth interventions should consider investigating the context under which mHealth could be more effective while considering its technical limitations in improving psychosocial health among pregnant women and mothers. Furthermore, future studies should also consider the psychosocial health of men transitioning into fatherhood and of same-sex and transgender partners.

Contributors Conceptualisation-JLS, RRC, MK, AS, EY, MB and MJ. Search strategy-JLS and RRC. Screening - JLS and RRC. Extraction and quality assessment-JLS, RRC and MK. Analysis and interpretation of data-JLS, RRC, MK, AS and MJ. Drafting the manuscript-JLS. Revising the manuscript-JLS, RRC, MK, AS and MJ. Guarantor-MJ. All authors critically reviewed and approved the manuscript.

Funding This research was funded by Program of Bilateral Health and Medical Cooperation between Japan and Russian Federation, Ministry of Health, Labor, and Welfare, Japan.

Competing interests None declared.

Patient consent for publication Not required.

Ethics approval This study does not involve human participants.
Provenance and peer review Not commissioned; externally peer reviewed.

Data availability statement All data relevant to the study are included in the article or uploaded as supplemental information.

Supplemental material This content has been supplied by the author(s). It has not been vetted by BMJ Publishing Group Limited (BMJ) and may not have been peer-reviewed. Any opinions or recommendations discussed are solely those of the author(s) and are not endorsed by BMJ. BMJ disclaims all liability and responsibility arising from any reliance placed on the content. Where the content includes any translated material, BMJ does not warrant the accuracy and reliability of the translations (including but not limited to local regulations, clinical guidelines, terminology, drug names and drug dosages), and is not responsible for any error and/or omissions arising from translation and adaptation or otherwise.

Open access This is an open access article distributed in accordance with the Creative Commons Attribution Non Commercial (CC BY-NC 4.0) license, which permits others to distribute, remix, adapt, build upon this work non-commercially, and license their derivative works on different terms, provided the original work is properly cited, appropriate credit is given, any changes made indicated, and the use is non-commercial. See: http://creativecommons.org/licenses/by-nc/4.0/.

\section{ORCID iDs}

Jennifer Lisa Sakamoto http://orcid.org/0000-0003-3444-1851

Rogie Royce Carandang http://orcid.org/0000-0001-6668-911X

Madhu Kharel http://orcid.org/0000-0003-0158-694X

Akira Shibanuma http://orcid.org/0000-0003-2058-1722

Masamine Jimba http://orcid.org/0000-0001-5659-3237

\section{REFERENCES}

1 Upton J. Psychosocial Factors. In: Encyclopedia of behavioral medicine, 2013: 1580-1.

2 Değirmenci F, Vefikuluçay Yılmaz D. The relationship between psychosocial health status and social support of pregnant women. $J$ Psychosom Obstet Gynaecol 2020;41:290-7.

3 Maxson PJ, Edwards SE, Valentiner EM, et al. A multidimensional approach to characterizing psychosocial health during pregnancy. Matern Child Health J 2016;20:1103-13.

4 Huizink AC, Menting B, De Moor MHM, et al. From prenatal anxiety to parenting stress: a longitudinal study. Arch Womens Ment Health 2017;20:663-72.

5 Walsh K, McCormack CA, Webster R, et al. Maternal prenatal stress phenotypes associate with fetal neurodevelopment and birth outcomes. Proc Natl Acad Sci U S A 2019;116:23996-4005.

6 Falah-Hassani K, Shiri R, Dennis C-L. Prevalence and risk factors for comorbid postpartum depressive symptomatology and anxiety. $J$ Affect Disord 2016;198:142-7.

7 Dennis C-L, Falah-Hassani K, Shiri R. Prevalence of antenatal and postnatal anxiety: systematic review and meta-analysis. $\mathrm{Br} \mathrm{J}$ Psychiatry 2017;210:315-23.

8 Bjelica A, Cetkovic N, Trninic-Pjevic A, et al. The phenomenon of pregnancy - a psychological view. Ginekol Pol 2018;89:102-6.

9 Shahhosseini Z, Pourasghar M, Khalilian A, et al. A review of the effects of anxiety during pregnancy on children's health. Mater Sociomed 2015;27:200-2.

10 Carmichael SL, Shaw GM. Maternal life event stress and congenital anomalies. Epidemiology 2000;11:30-5.

11 van den Heuvel JF, Groenhof TK, Veerbeek JH, et al. eHealth as the next-generation perinatal care: an overview of the literature. J Med Internet Res 2018;20:e202.

12 Hussain-Shamsy N, Shah A, Vigod SN, et al. Mobile health for perinatal depression and anxiety: Scoping review. J Med Internet Res 2020;22:e17011.

13 Klasnja P, Pratt W. Healthcare in the pocket: mapping the space of mobile-phone health interventions. J Biomed Inform 2012;45:184-98.

14 Chan KL, Chen M. Effects of social media and mobile health apps on pregnancy care: meta-analysis. JMIR Mhealth Uhealth 2019;7:e11836.

15 Lee Y, Moon M. Utilization and content evaluation of mobile applications for pregnancy, birth, and child care. Healthc Inform Res 2016;22:73-80

16 Dansharif AR, lyawa GE, Owoseni $A$, et al. mHealth for selfmanagement in pregnancy: perceptions of women in low-resource settings. Procedia Comput Sci 2021;181:738-45.

17 Tumuhimbise W, Atukunda EC, Ayebaza S, et al. Maternal healthrelated barriers and the potentials of mobile health technologies: 
qualitative findings from a pilot randomized controlled trial in rura southwestern Uganda. J Family Med Prim Care 2020;9:3657-62.

18 Relyea B, Wringe A, Afaneh O, et al. Stakeholders' perspectives on the challenges of emergency obstetric referrals and the feasibility and acceptability of an mHealth intervention in northern Iraq. Front Glob Womens Health 2021;2:662256.

19 Wang N, Deng Z, Wen LM, et al. Understanding the use of smartphone apps for health information among pregnant Chinese women: mixed methods study. JMIR Mhealth Uhealth 2019;7:e12631.

20 Dol J, Richardson B, Murphy GT, et al. Impact of mobile health interventions during the perinatal period on maternal psychosocial outcomes: a systematic review. JBI Evid Synth 2020;18:30-55.

21 Chen H, Chai Y, Dong L, et al. Effectiveness and appropriateness of mHealth interventions for maternal and child health: systematic review. JMIR Mhealth Uhealth 2018;6:e7.

22 Overdijkink SB, Velu AV, Rosman AN, et al. The usability and effectiveness of mobile health technology-based lifestyle and medical intervention apps supporting health care during pregnancy: systematic review. JMIR Mhealth Uhealth 2018;6:e109.

23 Mbuthia F, Reid M, Fichardt A. mHealth communication to strengthen postnatal care in rural areas: a systematic review. BMC Pregnancy Childbirth 2019;19:406.

24 Feroz A, Perveen S, Aftab W. Role of mHealth applications for improving antenatal and postnatal care in low and middle income countries: a systematic review. BMC Health Serv Res 2017;17:704.

25 Kabongo EM, Mukumbang FC, Delobelle P, et al. Explaining the impact of mHealth on maternal and child health care in low- and middle-income countries: a realist synthesis. BMC Pregnancy Childbirth 2021;21:196.

26 Dol J, Richardson B, Tomblin Murphy G, et al. Impact of mobile health (mHealth) interventions during the perinatal period for mothers in low- and middle-income countries: a systematic review. JBI Database System Rev Implement Rep 2019;17:1634-67.

27 Moher D, Liberati A, Tetzlaff J, et al. Preferred reporting items for systematic reviews and meta-analyses: the PRISMA statement. PLoS Med 2009;6:e1000097.

28 Cochrane. Rob 2: a revised Cochrane Risk-of-Bias tool for randomized trials. Available: https://methods.cochrane.org/bias/ resources/rob-2-revised-cochrane-risk-bias-tool-randomized-trials [Accessed 18 Jun 2021].

29 Cochrane. ROBINS-I tool. Available: https://methods.cochrane.org/ methods-cochrane/robins-i-tool [Accessed 18 Jun 2021].

30 National Heart, Lung, and Blood Institute. Quality assessment tool for observational cohort and cross-sectional studies. Available: https:// www.nhlbi.nih.gov/health-topics/study-quality-assessment-tools [Accessed 18 Jun 2021]

31 CASP Qualitative Studies Checklist. Critical appraisal skills programme. Available: https://casp-uk.net/casp-tools-checklists/ [Accessed 18 Jun 2021]

32 Hong QN, Gonzalez-Reyes A, Pluye P. Improving the usefulness of a tool for appraising the quality of qualitative, quantitative and mixed methods studies, the mixed methods appraisal tool (MMAT). J Eval Clin Pract 2018;24:459-67.

33 Granholm A, Alhazzani W, Møller MH. Use of the grade approach in systematic reviews and guidelines. Br J Anaesth 2019;123:554-9.

34 Campbell M, McKenzie JE, Sowden A, et al. Synthesis without meta-analysis (swim) in systematic reviews: reporting guideline. BMJ 2020;368:16890.

35 Bogale B, Mørkrid K, Abbas E, et al. The effect of a digital targeted client communication intervention on pregnant women's worries and satisfaction with antenatal care in Palestine-A cluster randomized controlled trial. PLoS One 2021;16:e0249713.

36 Cheng P-J, Wu T-L, Shaw S-W, et al. Anxiety levels in women undergoing prenatal maternal serum screening for Down syndrome: the effect of a fast reporting system by mobile phone short-message service. Prenat Diagn 2008;28:417-21.

37 Choi J, Lee JH, Vittinghoff E, et al. mHealth physical activity intervention: a randomized pilot study in physically inactive pregnant women. Matern Child Health J 2016;20:1091-101.

38 Chyzzy B, Nelson LE, Stinson J, et al. Adolescent mothers' perceptions of a mobile Phone-Based peer support intervention. Can J Nurs Res 2020;52:129-38.

39 Constant D, de Tolly K, Harries J, et al. Mobile phone messages to provide support to women during the home phase of medical abortion in South Africa: a randomised controlled trial. Contraception 2014;90:226-33.

40 Dennis-Tiwary TA, Denefrio S, Gelber S. Salutary effects of an attention bias modification mobile application on biobehavioral measures of stress and anxiety during pregnancy. Biol Psychol 2017;127:148-56.
41 Gallegos D, Russell-Bennett R, Previte J, et al. Can a text message a week improve breastfeeding? BMC Pregnancy Childbirth 2014; $14: 374$

42 Garfield CF, Lee YS, Kim HN, et al. Supporting parents of premature infants transitioning from the NICU to home: a pilot randomized control trial of a smartphone application. Internet Interv 2016;4:131-7.

43 Hannan J, Brooten D, Page T, et al. Low-Income first-time mothers: effects of APN follow-up using mobile technology on maternal and infant outcomes. Glob Pediatr Health 2016;3:1-10.

44 Hantsoo L, Criniti S, Khan A, et al. A mobile application for monitoring and management of depressed mood in a vulnerable pregnant population. Psychiatr Serv 2018;69:104-7.

45 Jannati N, Mazhari S, Ahmadian L, et al. Effectiveness of an app-based cognitive behavioral therapy program for postpartum depression in primary care: a randomized controlled trial. Int $J$ Med Inform 2020;141:104145.

46 Jareethum R, Titapant V, Chantra T, et al. Satisfaction of healthy pregnant women receiving short message service via mobile phone for prenatal support: a randomized controlled trial. J Med Assoc Thai 2008;91:458-63.

47 Kodama T, Obayashi Y, Tanimura S, et al. A randomized controlled trial on primigravid women of text messaging intervention offering pregnancy and childbirth support. $J$ Uoeh 2021;43:305-12.

48 Seyyedi N, Rahmatnezhad L, Mesgarzadeh M, et al. Effectiveness of a smartphone-based educational intervention to improve breastfeeding. Int Breastfeed J 2021;16:70.

49 Shorey S, Lau Ying/Y, Dennis C-L, et al. A randomized-controlled trial to examine the effectiveness of the 'Home-but not Alone' mobilehealth application educational programme on parental outcomes. $J$ Adv Nurs 2017;73:2103-17.

50 Sun Y, Li Y, Wang J, et al. Effectiveness of smartphone-based mindfulness training on maternal perinatal depression: randomized controlled trial. J Med Internet Res 2021;23:e23410.

51 Takeuchi S, Horiuchi S. Randomised controlled trial using smartphone website vs leaflet to support antenatal perineal massage practice for pregnant women. Women Birth 2016;29:430-5.

52 McGuinness LA, Higgins JPT. Risk-of-bias visualization (robvis): an R package and shiny web APP for visualizing risk-of-bias assessments. Res Synth Methods 2021;12:55-61.

53 Carissoli C, Gasparri D, Riva G. Mobile well-being in pregnancy: suggestions from a quasi-experimental controlled study. Behav Inf Technol 2021.

54 Dalton JA, Rodger D, Wilmore M, et al. The Health-e babies APP for antenatal education: feasibility for socially disadvantaged women. PLoS One 2018;13:e0194337.

55 Song $\mathrm{H}$, May A, Vaidhyanathan V, et al. A two-way text-messaging system answering health questions for low-income pregnant women. Patient Educ Couns 2013;92:182-7.

56 Trude ACB, Martins RC, Martins-Silva T, et al. A WhatsApp-based intervention to improve maternal social support and maternalchild health in southern Brazil: the text-message intervention to enhance social support (ties) feasibility study. Inquiry 2021;58:469580211048701.

57 Abroms LC, Johnson PR, Heminger CL, et al. Quit4baby: results from a pilot test of a mobile smoking cessation program for pregnant women. JMIR Mhealth Uhealth 2015;3:e10.

58 Fujioka N, Kobayashi T, Turale S. Short-Term behavioral changes in pregnant women after a quit-smoking program via e-learning: a descriptive study from Japan. Nurs Health Sci 2012;14:304-11.

59 Jallo N, Thacker LR, Menzies V, et al. A stress coping APP for hospitalized pregnant women at risk for preterm birth. MCN Am J Matern Child Nurs 2017;42:257-62.

60 Kubo A, Aghaee S, Kurtovich EM, et al. mHealth mindfulness intervention for women with Moderate-to-Moderately-Severe antenatal depressive symptoms: a pilot study within an integrated health care system. Mindfulness 2021:1-11.

61 Baumel A, Tinkelman A, Mathur N, et al. Digital peer-support platform (7Cups) as an adjunct treatment for women with postpartum depression: feasibility, acceptability, and preliminary efficacy study. JMIR Mhealth Uhealth 2018;6:e38.

62 Globus O, Leibovitch L, Maayan-Metzger A, et al. The use of short message services (SMS) to provide medical updating to parents in the NICU. J Perinatol 2016;36:739-43.

63 Goetz M, Schiele C, Müller M, et al. Effects of a brief electronic mindfulness-based intervention on relieving prenatal depression and anxiety in hospitalized high-risk pregnant women: exploratory pilot study. J Med Internet Res 2020;22:e17593.

64 Deave T, Ginja S, Goodenough T, et al. The bumps and babies longitudinal study (BaBBLeS): a multi-site cohort study of first- 
time mothers to evaluate the effectiveness of the baby Buddy APP. Mhealth 2019;5:42.

65 Özkan Şat S, Yaman Sözbir Şengül. Use of mobile applications and blogs by pregnant women in turkey and the impact on adaptation to pregnancy. Midwifery 2018;62:273-7.

66 Brown S, Brage Hudson D, Campbell-Grossman C, et al. Health promotion text blasts for minority adolescent mothers. MCN Am J Matern Child Nurs 2014;39:357-62.

67 Connor K, Wambach K, Baird MB. Descriptive, qualitative study of women who use mobile health applications to obtain perinatal health information. J Obstet Gynecol Neonatal Nurs 2018;47:728-37.

68 Harrington EK, McCoy EE, Drake AL, et al. Engaging men in an $\mathrm{mHealth}$ approach to support postpartum family planning among couples in Kenya: a qualitative study. Reprod Health 2019;16:17.

69 Litterbach E-K, Russell CG, Taki S, et al. Factors influencing engagement and behavioral determinants of infant feeding in an mHealth program: qualitative evaluation of the growing healthy program. JMIR Mhealth Uhealth 2017;5:e196.

70 Seshu U, Khan HA, Bhardwaj M, et al. A qualitative study on the use of mobile-based intervention for perinatal depression among perinatal mothers in rural Bihar, India. Int $J$ Soc Psychiatry 2021;67:467-71.

71 Skar JB, Garnweidner-Holme LM, Lukasse M, et al. Women's experiences with using a smartphone APP (the Pregnant+ APP) to manage gestational diabetes mellitus in a randomised controlled trial. Midwifery 2018;58:102-8.

72 Yee L, Taylor S, Young M, et al. Evaluation of a text messaging intervention to support self-management of diabetes during pregnancy among low-income, minority women: qualitative study. JMIR Diabetes 2020;5:e17794.

73 Avalos LA, Aghaee S, Kurtovich E, et al. A mobile health mindfulness intervention for women with moderate to moderately severe postpartum depressive symptoms: feasibility study. JMIR Ment Health 2020;7:e17405.

74 Blackwell TM, Dill LJ, Hoepner LA, et al. Using text messaging to improve access to prenatal health information in urban African American and Afro-Caribbean immigrant pregnant women: mixed methods analysis of Text4baby usage. JMIR Mhealth Uhealth 2020;8:e14737.

75 Musiimenta A, Tumuhimbise W, Pinkwart N, et al. A mobile phonebased multimedia intervention to support maternal health is acceptable and feasible among illiterate pregnant women in Uganda: qualitative findings from a pilot randomized controlled trial. Digit Health 2021;7:205520762098629.

76 Rhodes A, Kheireddine S, Smith AD. Experiences, attitudes, and needs of users of a pregnancy and parenting APP (baby Buddy) during the COVID-19 pandemic: mixed methods study. JMIR Mhealth Uhealth 2020;8:e23157.
77 Seo JM, Kim S-J, Na H, et al. The development of the postpartum depression self-management mobile application "Happy Mother". Comput Inform Nurs 2021;39:439-49.

78 Simpson N, Kydd A, Phiri M, et al. Insaka: mobile phone support groups for adolescent pregnant women living with HIV. BMC Pregnancy Childbirth 2021;21:663.

79 Stonbraker S, Haight E, Lopez A, et al. Digital educational support groups administered through WhatsApp messenger improve healthrelated knowledge and health behaviors of new adolescent mothers in the Dominican Republic: a multi-method study. Informatics 2020;7:51.

80 The World Bank. World bank country and lending groups. Available: https://datahelpdesk.worldbank.org/knowledgebase/articles/906519world-bank-country-and-lending-groups [Accessed 24 Jun 2021].

81 Labrique $A B$, Vasudevan $L$, Kochi $E$, et al. mHealth innovations as health system strengthening tools: 12 common applications and a visual framework. Glob Health Sci Pract 2013;1:160-71.

82 Kosugi H, Shibanuma A, Kiriya J, et al. Consistent condom use among highly effective contraceptive users in an HIV-endemic area in rural Kenya. PLoS One 2019;14:e0216208.

83 Håkansson M, Super S, Oguttu M, et al. Social judgments on abortion and contraceptive use: a mixed methods study among secondary school teachers and student peer-counsellors in Western Kenya. BMC Public Health 2020;20:493.

84 Jackson L, De Pascalis L, Harrold J, et al. Guilt, shame, and postpartum infant feeding outcomes: a systematic review. Matern Child Nutr 2021;17:e13141.

85 Oh S, Chew-Graham CA, Silverwood V, et al. Exploring women's experiences of identifying, negotiating and managing perinatal anxiety: a qualitative study. BMJ Open 2020;10:e040731.

86 Bhatt J, Bathija P. Ensuring access to quality health care in vulnerable communities. Acad Med 2018;93:1271-5.

87 Kruse C, Betancourt J, Ortiz S, et al. Barriers to the use of mobile health in improving health outcomes in developing countries: systematic review. J Med Internet Res 2019;21:e13263.

88 Grossman LV, Masterson Creber RM, Benda NC, et al. Interventions to increase patient portal use in vulnerable populations: a systematic review. J Am Med Inform Assoc 2019;26:855-70.

89 Armaou M, Araviaki E, Musikanski L. eHealth and mHealth interventions for ethnic minority and historically underserved populations in developed countries: an umbrella review. Int $J$ Community Wellbeing 2020;3:193-221.

90 Giurgescu C, Zenk SN, Templin TN, et al. The impact of neighborhood environment, social support, and avoidance coping on depressive symptoms of pregnant African-American women. Womens Health Issues 2015;25:294-302.

91 Gurupur VP, Wan TTH. Challenges in implementing mHealth interventions: a technical perspective. Mhealth 2017;3:32. 